\title{
Introduction: Endovascular Neurosurgery
}

\author{
Elad I. Levy, MD, MBA, FACS, FAHA,1-4 Stephan A. Munich, MD,,1,2 Robert H. Rosenwasser, MD, MBA, \\ FACS, FAHA, ${ }^{5}$ Peter Kan, MD, MPH, FAANS, FRCS(C), ${ }^{6}$ and B. Gregory Thompson, MD, FAANS ${ }^{7}$
}

${ }^{1}$ Department of Neurosurgery, Gates Vascular Institute at Kaleida Health, Buffalo; Departments of ${ }^{2}$ Neurosurgery and ${ }^{3}$ Radiology, Jacobs School of Medicine and Biomedical Sciences, University at Buffalo; ${ }^{4}$ Canon Stroke and Vascular Research Center, University at Buffalo, New York; ${ }^{5}$ Department of Neurological Surgery, Thomas Jefferson University and Jefferson Hospital for Neuroscience, Philadelphia, Pennsylvania; ${ }^{6}$ Department of Neurosurgery, Baylor College of Medicine, Houston, Texas; and ${ }^{7}$ Department of Neurosurgery, University of Michigan, Ann Arbor, Michigan

Remarkable developments in the field of endovascular neurosurgery have been witnessed in the last decade. The success of endovascular therapy for ischemic stroke treatment is now irrefutable, making it an accepted standard of care. Endovascular treatment of cerebral aneurysms is no longer limited to primary coiling but now includes options such as stent or balloon assistance and flow diversion and applications utilizing neck reconstruction, intrasaccular, and bifurcation-specific devices. Balloons, liquid embolic agents, and flow-directed catheters have revolutionized the treatment of arteriovenous malformations and fistulae. The ongoing development of endovascular tools has led to novel and expanding approaches (for example, transvenous arteriovenous malformation embolization and transradial access). With improved technology, transposterior communicating artery access and other endovascular strategies are being applied successfully across the anterior and posterior circulations and to lesions once deemed only surgically approachable. Yet, we would be remiss to attribute the successes of endovascular strategies only to the development of their tools. Improvements in both noninvasive and angiographic imaging (such as three-dimensional road map guidance) have provided a greater understanding of pathologic entities and allowed the pursuit of endovascular cures.

In this issue of Neurosurgical Focus, we present a wide range of endovascular strategies for a variety of neurovascular pathologies. We hope this video supplement will not only demonstrate the applicability of tried-and-true endovascular strategies to difficult clinical situations but also highlight new and developing endovascular technologies. We thank the authors for their outstanding contributions.

KEYWORDS endovascular neurosurgery; neurovascular pathologies; technique; technology; video 